\title{
VDI
}

\section{REIHE 23}

TECHNISCHE GEBÄUDEAUSRÜSTUNG

FortschrittBerichte VDI

Dipl.-Ing. (FH) René Stang, M.Eng.; Kutzleben

NR. 6

\section{Methode zur Ökoeffizienzbewertung wärmetechnischer Anlagen in Gebäuden}

BAND

$1 \mid 1$

VOLUME 
https://doi.org/10.51202/9783186006233-

Generiert durch IP '172.22.53.54', am 26.04.2023, 04:17:12.

Das Erstellen und Weitergeben von Kopien dieses PDFs ist nicht zulässig. 


\section{Methode zur Ökoeffizienzbewertung wärmetechnischer Anlagen in Gebäuden}

Ein Beitrag zur ganzheitlichen Nachhaltigkeit

Von der Fakultät Architektur und Urbanistik der Bauhaus-Universität Weimar

zur Erlangung des akademischen Grades

Doktor-Ingenieur (Dr.-Ing.)

genehmigte

\section{Dissertation}

vorgelegt von

Dipl.-Ing. (FH) René Stang, M.Eng.

Gutachter: Prof. Dr.-Ing. Bernd Nentwig

Prof. Dr.-Ing. Prof. h.c. Jens Mischner

Prof. Dr.-Ing. Hartmut Krause

Tag der Disputation: 30. April 2021 
https://doi.org/10.51202/9783186006233-

Generiert durch IP '172.22.53.54', am 26.04.2023, 04:17:12.

Das Erstellen und Weitergeben von Kopien dieses PDFs ist nicht zulässig. 


\section{VDI}

REIHE 23

TECHNISCHE GEBÄUDEAUSRÜSTUNG

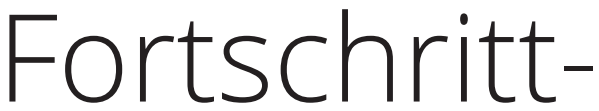
Berichte VDI

Dipl.-Ing. (FH) René Stang, M.Eng.; Kutzleben

NR. 6
Methode zur
Ökoeffizienzbewertung
wärmetechnischer
Anlagen in Gebäuden

BAND

1|1

VOLUME 


\section{VDI verlag}

Stang, René

\section{Methode zur Ökoeffizienzbewertung wärmetechnischer Anlagen in Gebäuden}

Fortschritt-Berichte VDI, Reihe 23, Nr. 6. Düsseldorf: VDI Verlag 2021.

212 Seiten, 82 Bilder, 24 Tabellen.

ISBN 978-3-18-300623-6, ISSN 2192-6727

76,00 EUR/VDI-Mitgliederpreis: 68,40 EUR

Für die Dokumentation: Energiewirtschaft - Nachhaltigkeitsbewertung - Ökobilanz - Ökoeffizienz - Primärenergie - Primärenergiefaktor - regenerative Energien - Umweltleistung - Wärmetechnik - Wirtschaftlichkeit

Keywords: energy supply chain - energy industry - sustainability assessment - life cycle assessment eco-efficiency - primary energy - primary energy factor - renewable energies - environmental performance heat technology - economic efficiency

Die vorliegende Arbeit richtet sich an Ingenieur*innen und Wissenschaftler*innen der technischen Gebäudeausrüstung. Sie greift einen sich abzeichnenden Änderungsbedarf in der Umwelt- und Nachhaltigkeitsbewertung von Gebäuden und wärmetechnischen Anlagen auf. Der aktuell genutzte nicht erneuerbare Primärenergiebedarf wird insbesondere hinsichtlich künftiger politischer Klima- und Umweltschutzziele als alleinige Bewertungsgröße nicht ausreichend sein. Die mit dieser Arbeit vorgestellte Ökoeffizienzbewertungsmethode kann als geeignetes Instrument zur Lösung der Probleme beitragen. Sie ermöglicht systematische, ganzheitliche Bewertungen und reproduzierbare Vergleiche wärmetechnischer Anlagen bezüglich ihrer ökologischen und ökonomischen Nachhaltigkeit. Die wesentlichsten Neuentwicklungen sind die spezifische Umweltleistung, in Erweiterung zum genutzten Primärenergiefaktor, und der Ökoeffizienzindikator UWI.

\section{Bibliographische Information der Deutschen Bibliothek}

Die Deutsche Bibliothek verzeichnet diese Publikation in der Deutschen Nationalbibliographie;

detaillierte bibliographische Daten sind im Internet unter www.dnb.de abrufbar.

Bibliographic information published by the Deutsche Bibliothek (German National Library)

The Deutsche Bibliothek lists this publication in the Deutsche Nationalbibliographie

(German National Bibliography); detailed bibliographic data is available via Internet at www.dnb.de. 


\section{Vorwort}

Diese Dissertation entstand während meiner Tätigkeit als Angestellter der Fakultät Gebäudetechnik und Informatik an der Fachhochschule Erfurt sowie bei den Stadtwerken Jena in Kooperation mit der Bauhaus-Universität Weimar. Für die vielfältige Hilfe bei der Erstellung der Dissertation und das Gelingen des Promotionsvorhabens bin ich einer Reihe von Personen und Institutionen sehr dankbar.

Mein besonderer Dank gilt zunächst Herrn Prof. Dr.-Ing. Prof. h.c. Jens Mischner für die beharrliche, intensive Betreuung und verständnisvolle Unterstützung meiner Forschungsarbeit. Mein Dank gilt ebenso Herrn Prof. Dr.-Ing. Bernd Nentwig der dieses Promotionsvorhaben befürwortet und die Dissertation als Gutachter konstruktiv betreut hat. Weiterhin bedanke ich mich bei Herrn Prof. Dr.-Ing. Hartmut Krause für die Begutachtung meiner Arbeit.

Ich bedanke mich darüber hinaus bei allen Kollegen der Fachrichtung Gebäude- und Energietechnik der Fachhochschule Erfurt für ihre fortwährende Unterstützung bei der Umsetzung meines Vorhabens. Besonders erwähnen möchte ich Herrn Dipl.-Ing. (FH) Uwe Sandner für die stets wertvollen und tiefgreifenden Diskussionen über die Forschungsarbeit. Auch seien Frau Prof. Dr.-Ing. Cornelia König und Herr Prof. Dr.-Ing. Michael Kappert genannt, die als Leitende der Fachrichtung jederzeit förderliche Hinweise und Hilfestellungen zur Umsetzung meiner Arbeit gegeben haben.

Ebenso bin ich der Stadtwerke Jena Netze GmbH, die meine Dissertation besonders gefördert hat, zu großem Dank verpflichtet.

Nicht zuletzt empfinde ich tiefe Dankbarkeit gegenüber meiner lieben Frau Karina sowie unseren Kindern Johanna und Theo, ohne deren Unterstützung und Verständnis dieses Promotionsvorhaben nicht gelungen wäre. 
Wir haben die Erde nicht von unseren Vorfahren geerbt, wir haben sie von unseren Nachkommen geliehen.

Alte indianische Weisheit 


\section{Inhaltsverzeichnis}

Kurzfassung VIII

Abstract $\quad X$

Nomenklatur XII

1 Einführung $\quad 1$

1.1 Problemstellung . . . . . . . . . . . . . . . . . . . . . . . . . 1

1.2 Zielsetzung . . . . . . . . . . . . . . . . . . . . 3

1.3 Herangehensweise ... . . . . . . . . . . . . . . . . . 4

1.4 Aufbau der Arbeit . . . . . . . . . . . . . . . . . . . . . . 6

2 Nachhaltigkeitsbewertung in Wissenschaft und Technik 7

2.1 Lebenszyklusbasierte Nachhaltigkeitsbewertung . . . . . . . . . . . . . . . 7

2.1.1 Nachhaltigkeitsgedanke . . . . . . . . . . . . . . . . 7

2.1.2 Nachhaltigkeitsquantifizierung . . . . . . . . . . . . . . . . 10

2.2 Nachhaltigkeitsbewertung wärmetechnischer Anlagen . . . . . . . . . . . . 14

2.2.1 Primärenergetische Bewertung gemäß Gesetzgebung . . . . . . . . . 14

2.2.2 Auswahlkriterium Wirtschaftlichkeit . . . . . . . . . . . . . . . 19

2.3 Ökoeffizienzbewertung . . . . . . . . . . . . . . . . . . 21

2.3.1 Methodischer Bewertungsrahmen . . . . . . . . . . . . . . . . 21

2.3.2 Definition von Ziel und Untersuchungsrahmen . . . . . . . . . . . 24

2.3.3 Umweltbewertung mittels Ökobilanz . . . . . . . . . . . . . . . . . . . . 26

2.3.4 Monetärer Produktsystemnutzen . . . . . . . . . . . . . . . . . . 29

2.3.5 Ökoeffizienzquantifizierung . . . . . . . . . . . . . . . . . . . . . . . . . . . . . .

2.3 .6 Auswertung . . . . . . . . . . . . . . . . . 32

3 Methodenentwicklung $\quad 34$

3.1 "Erfurter Methode" . . . . . . . . . . . . . . . . . . . . . . . . 34

3.1 Bewertungskonzept ... . . . . . . . . . . . . 34

3.1 .2 Bewertungsablauf . . . . . . . . . . . . . . . . . . 37

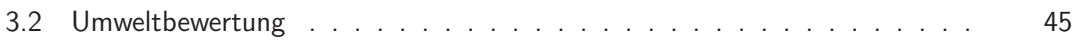

3.2.1 Vorgehensweise. . . . . . . . . . . . . . . . 45

3.2 .2 Umweltleistung . . . . . . . . . . . . . . . . . . . . . . . . . . . . 46

3.2 .2 .1 Definition . . . . . . . . . . . . . . 46 
3.2.2.2 Anlagenkomponenten ............... . . 49

3.2.2.3 Endenergieträger . . . . . . . . . . . . . . 62

3.2.3 Umweltleistungsindikator . . . . . . . . . . . . . . . . . 75

3.2.4 Spezifische Umweltleistung . . . . . . . . . . . . . . . . 75

3.2.4.1 Definition . . . . . . . . . . . . . . . 75

3.2.4.2 Sachbilanz . . . . . . . . . . . . . . . . 76

3.2.4.3 Wirkungsabschätzung ............... 86

3.2 .5 Unsicherheitsanalyse . . . . . . . . . . . . . . . . . . . . . 98

3.2.5.1 Unsicherheit . . . . . . . . . . . . . . . . . . . . . . 98

3.2.5.2 Standardfehler der Regression . . . . . . . . . . . . . 101

3.2.5.3 Stichprobenstandardabweichung . . . . . . . . . . 102

3.3 Wirtschaftlichkeitsbewertung . . . . . . . . . . . . . 106

3.3 .1 Vorgehensweise . . . . . . . . . . . . . . . . . . 106

3.3.2 Wirtschaftlichkeit ... . . . . . . . . . . . . . 109

3.3.2.1 Definition . . . . . . . . . . . . . . . . 109

3.3.2.2 Anlagenkomponenten . . . . . . . . . . . . . . 109

3.3.2.3 Endenergieträger . . . . . . . . . . . . . . . . . . 113

3.3.3 Wirtschaftlichkeitsindikator . . . . . . . . . . . . . . . . 119

3.4 Ökoeffizienzbewertung . . . . . . . . . . . . . . . . . . . . . . . . 119

3.4 .1 Ökoeffizienz . . . . . . . . . . . . . . . . . . . . . . . 119

3.4 .2 Ökoeffizienzindikator . . . . . . . . . . . . . . . . . . . 120

3.4.3 Ökoeffizienznomogramm . . . . . . . . . . . . . . . . 121

4 Methodenanwendung $\quad 127$

4.1 Bewertungsszenario . . . . . . . . . . . . . . . . . . . . . . . 127

4.1.1 Zielsetzung und Untersuchungsrahmen . . . . . . . . . . . . 127

4.1.2 Umwelt- und Wirtschaftlichkeitsbewertung . . . . . . . . . . . . . 129

4.2 Ergebnisinterpretation . . . . . . . . . . . . . . . . . 130

4.2.1 Umweltbewertung . . . . . . . . . . . . . . . . . . . . 130

4.2.2 Wirtschaftlichkeitsbewertung . . . . . . . . . . . . . . 132

4.2 .3 Ökoeffizienzbewertung . . . . . . . . . . . . . . . . . . . . 133

4.2 .4 Unsicherheitsanalyse . . . . . . . . . . . . . . . . . . . . . 134

$\begin{array}{lll}5 & \text { Zusammenfassung } & 140\end{array}$

5.1 Fazit . . . . . . . . . . . . . . . . . . . . . 140

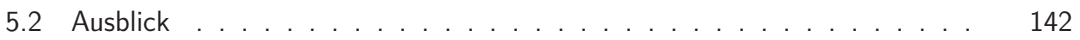

Anhang A Ergänzungen zur Nachhaltigkeitsbewertung $\quad 145$

A.1 Lebenszyklusbasierte Nachhaltigkeitsbewertungsmethoden . . . . . . . . 145

A.2 Methodenauswahl . . . . . . . . . . . . . . . . . 145 
$\begin{array}{ll}\text { Anhang B Ergänzungen zur Methodenentwicklung } & 148\end{array}$

B.1 Berechnung der Skalierfaktoren . . . . . . . . . . . . . . . . 148

B.2 Spezifische Umweltleistung und deren Unsicherheit . . . . . . . . . . . . . 148

B.3 Sachbilanzpläne der Anlagenkomponente Fußbodenheizung . . . . . . . . 151

B.4 Datenbanken zur Ökobilanzierung . . . . . . . . . . . . . . . . . 160

B.5 Korrektur des Fernwärmemix . . . . . . . . . . . . . 166

$\begin{array}{ll}\text { Anhang C Ergänzungen zur Methodenanwendung } & 170\end{array}$

C.1 Energetische Bewertung . . . . . . . . . . . . . . . . . . . . 170

C.2 Unsicherheitsanalyse . . . . . . . . . . . . . . . . . . . . 170

$\begin{array}{lr}\text { Literatur } & 178\end{array}$ 


\section{Kurzfassung}

Die vorliegende Dissertation greift eine aktuell zur Diskussion gestellte und sich abzeichnende Änderung in der Umwelt- und Nachhaltigkeitsbewertung von Gebäuden und wärmetechnischen Anlagen sowie deren Endenergieträgern auf. Es ist zu vermuten, dass die gegenwärtige Verfahrensweise gemäß Energieeinsparverordnung (EnEV) künftig zu praktischen Problemen führt und eine Neuausrichtung erforderlich ist. Der nichterneuerbare Primärenergiebedarf wird in Zukunft nicht als alleinige Umweltbewertungsgröße für die Auswahl wärmetechnischer Anlagen sowie deren Endenergieträger ausreichend sein. Dies gilt besonders für die Umsetzung bevorstehender politischer Klima- und Umweltschutzanforderungen, wie die weitere Reduzierung von Treibhausgasen oder Ressourceneinsparungen.

Die Herausforderung für Systemplaner besteht somit zunehmend in der Aufgabe, ganzheitlich nachhaltige Anlagen zu konzipieren und zu errichten. In diesem Zusammenhang müssen die Akteure mehr oder weniger bewusst die Frage beantworten, welche mögliche Anlagenvariante für ein gegebenes Gebäude die gestellten Nachhaltigkeitskriterien im Zusammenwirken mit den baulichen Wärmeschutzmaßnahmen optimal erfüllt. Um diese komplexe Aufgabenstellung zu lösen sowie eine umfängliche Beurteilung wärmetechnischer Anlagen hinsichtlich ihrer Umweltbeeinflussung und Nachhaltigkeit vornehmen zu können, sind neue, erweiterte und ganzheitliche Bewertungsinstrumente notwendig. Mit Blick auf die Problemstellung sowie den aktuellen Stand der Wissenschaft und Technik wurde das primäre Ziel dieser Forschungsarbeit formuliert. Es sollte ein geeignetes quantitatives Bewertungsinstrument zur Ökoeffizienzbewertung wärmetechnischer Anlagen entwickelt und bereitgestellt werden.

In dieser Dissertation wird unter dem Arbeitstitel „Erfurter Methode” eine mögliche Lösung vorgeschlagen. Sie verknüpft ökologische und ökonomische Größen zu einem gemeinsamen Nachhaltigkeitsindikator. Die Beurteilung erfolgt ganzheitlich über den gesamten Lebensweg. Für die Umweltbewertung und die Aggregation der Bewertungsgrößen zu einem Vergleichsindikator mussten neue Ansätze entwickelt werden. Mit der vorliegenden Umweltbewertungsmethode und der zugehörigen Umweltleistungsermittlung der Anlagenkomponenten stellt die Methode gegenüber dem Primärenergieverfahren eine zukunftsweisende Erweiterung dar. Zur ökonomischen Beurteilung konnte hingegen auf bekannte Verfahren der Wirtschaftlichkeitsberechnung zurückgegriffen werden. Insbesondere mit dem Ökoeffizienzindikator als vollaggregiertem Vergleichsindikator ist die quantitative Verknüpfung ökologischer und ökonomischer Nachhaltigkeitsbewertungsergebnisse möglich. Er wird zudem genutzt, um bei mehreren wärmetechnischen Anlagenvarianten für ein Gebäude die vorteilhafteste (ökoeffizienteste) Lösung zu finden. Zur 
einfachen Ergebnisinterpretation wurde zusätzlich ein Ökoeffizienznomogramm entwickelt. Es stellt die Resultate der Umwelt-, Wirtschaftlichkeits- und Ökoeffizienzbewertung mehrerer Anlagenvarianten gegenüber und ist für deren Vergleich nutzbar.

Künftig soll die „Erfurter Methode” auch auf andere gebäudetechnische Anlagen (Sanitär-, Klimatechnik) und das gesamte Gebäude erweitert werden. Ebenso ist die Bewertung sozialer Aspekte mit dem Ziel einer ganzheitlichen Nachhaltigkeitsbeurteilung angedacht. 


\section{Abstract}

This dissertation deals with a currently discussed and emerging change in the environmental and sustainability assessment of buildings, heating systems and their final energy sources. Presumably, the current procedure according to the Germany Energy Saving Ordinance (EnEV) will lead to future practical problems and a realignment will be necessary. In the future, the nonrenewable primary energy demand will not be sufficient as the only environmental assessment value for the selection of heating systems and their final energy sources. This applies particularly to the implementation of upcoming political climate and environmental protection requirements, such as the further reduction of greenhouse gases or resource savings.

Therefore, the challenge for system planners is increasingly to design and build holistically sustainable installations. In this context, the participants have to answer more or less consciously the question of which system variant for a given building best meets the sustainability requirements in conjunction with the structural thermal protection measures. In order to solve this complex task and to carry out a comprehensive assessment of heating systems with regard to their environmental impact and sustainability, new expanded and holistic assessment instruments are needed. The primary goal of this research work was formulated in view of the problem and the current state of science and technology. A suitable quantitative assessment tool for the eco-efficiency assessment of heating systems had to be developed and made available.

In this dissertation, a possible solution is proposed under the working title "Erfurt Method". It combines ecological and economic parameters into a joint sustainability indicator. The assessment considers the entire life cycle. New approaches had to be developed for the environmental assessment and the aggregation of the assessment parameters into a single comparative indicator. With the present environmental assessment method and the associated environmental performance determination of system components, the procedure represents a forward-looking extension compared to the primary energy method. For the economic assessment, however, known methods of profitability analysis could be used. The eco-efficiency indicator in particular, as a fully aggregated comparison indicator, enables the quantitative combination of ecological and economic sustainability assessment results. It is also used to find the most favorable (eco-efficient) solution for a building from several heating systems. In addition, an eco-efficiency nomogram was developed for easy interpretation of the outcome. It shows the results of the environmental, economic and eco-efficiency assessment of the system variants and can be used for their comparison. 
In the future, the "Erfurt Method" is to be expanded to include other building technology systems (sanitary, air conditioning) and the entire building. The evaluation of social aspects, aiming at a holistic sustainability assessment, is also being considered. 


\section{Nomenklatur}

\section{Abkürzungen}

AA

AGFW

$\mathrm{AL}$

BHKW

BNB

BREEAM

BW

CF

$\mathrm{CH}_{4}$

CLR

$\mathrm{CO}_{2}$

DALY

DE

DGNB

ED

EEA
Additional Aspects, zusätzliche Aspekte

Arbeitsgemeinschaft Fernwärme, Energieeffizienzverband für Wärme, Kälte und KWK e.V.

Aluminium

Blockheizkraftwerk

Bewertungssystem Nachhaltiges Bauen für Bundesgebäude

Building Research Establishment Environmental Assessment Method

Brennwertkessel

Carbon Footprint, $\mathrm{CO}_{2}$-Fußabdruck

Methan

Closed-Loop Recycling, Recycling im geschlossenen Kreislauf

Kohlenstoffdioxid

Disability-adjusted loss of life years, Lebenszeitverlust aufgrund von Krankheit, Behinderung und vorzeitigem Tod

Deutschland

Deutsche Gesellschaft für Nachhaltiges Bauen

Ecosystem Diversity, Ökosystemdiversität

Eco-Efficiency Analysis, Eco-Efficiency Assessment, Ökoeffizienzanalyse, Ökoeffizienzbewertung 
ELCA Environmental/Ecology Life Cycle Assessment, Ökobilanz

EM

Exergiemethode, Stromverlustmethode

EMAS Eco-Management and Audit Scheme, Umweltmanagement und Umweltbetriebsprüfung

Eol $\quad$ End of Life, Lebensende

EPD Environmental Product Declaration, Umweltproduktdeklaration

EPDM Ethylen-Propylen-Dien-Monomerkautschuk

EPS

expandiertes Polystyrol

EU

Europäische Union

FW

Fernwärme

GEG Gebäudeenergiegesetz, Gesetz zur Vereinheitlichung des Energieeinsparrechts für Gebäude

GEMIS Globales Emissions-Modell integrierter Systeme

GM Gutschriftenmethode

GuD Gas- und Dampfkraftwerk

GWP Global Warming Potential, Treibhauspotenzial

$\mathrm{HH} \quad$ Human Health, menschliche Gesundheit

HKW Heizkraftwerk

HS Hochspannung

HW Heizwerk

ILCSA Integrated Life Cycle Sustainability Assessment, integrierte lebenszyklusbasierte Nachhaltigkeitsbewertung

KEA kumulierter Energieaufwand

KWK Kraft-Wärme-Kopplung

LCA Life Cycle Assessment, Ökobilanz

LCC Life Cycle Costs, Lebenszykluskosten 
LCEA Life Cycle Environmental/Ecology Assessment, Ökobilanz

LCl Life Cycle Inventory, Sachbilanz

LCIA Life Cycle Impact Assessment, Wirkungsabschätzung

LCSA Life Cycle Sustainability Assessment, lebenszyklusbasierte Nachhaltigkeitsbewertung

LEED Leadership in Energy and Environmental Design

LPG Liquefied Petroleum Gas, Flüssiggas

m mit

MIPS Material-Input pro Serviceeinheit

MKWK Mikro-Kraft-Wärme-Kopplung

MS Mittelspannung

MöK Methode der ökologischen Knappheit

NS Niederspannung

o ohne

o.WG ohne Wärmegutschrift

OLR Open-Loop Recycling, Recycling im offenen Kreislauf

PE Primärenergie

PE-X vernetztes Polyethylen

PEF Product Envionmental Footprint, Umweltfußabdruck

PROSA Product Sustainability Assessment, Produktnachhaltigkeitsbewertung

RA Resource Availability, Ressourcenverfügbarkeit

$\mathrm{ReCiPe} \quad$ Akronym aus den Initialen der Organisationen (RIVM, Radboud University Nijmegen, Center of Environmental Sciences (CML, Leiden University), PRé Consultants), die diese Methode entwickelt haben

RNE Rat für Nachhaltige Entwicklung

SEEbalance Socio-Eco-Efficiency, Sozio-Ökoeffizienz-Bewertung 
SG

SLCA

TCO

TFA

ts

UBP

ULA

UVP

UZBP

VOFI

WBCSD

WE

WF

WG

WP

WTA

ZE

\section{Formelzeichen}

$\alpha$

$\beta$

$\Delta$

Differenz

thinkstep de"
Standgerät

Social Life Cycle Assessment, Sozialbilanz

Total Cost of Ownership, Gesamtbetriebskosten

Technikfolgenabschätzung

Umweltbelastungspunkte, Einheit der bewerteten Umweltwirkung nach der UBP-Methode

Umweltleistungsanteil, Einheit der Umweltleistung nach der „Erfurter Metho-

Umweltverträglichkeitsprüfung

Umweltzielbelastungspunkte

vollständiger Finanzplan

World Business Council for Sustainable Development

Wohneinheiten

Water Footprint, Wasser-Fußabdruck

Wandgerät

Wärmepumpe

wärmetechnische Anlage

Zementestrich

Anteil, Aufteilungsfaktor

Jahresheizwärme-, Energiebedarfs-, Energieanteil, spezifische Exergie, Stromverlustkoeffizient 
$\eta$

$\hat{Y}$

(D)

$\rho$

$\sigma$

$\varepsilon$

$\vartheta$

$\dot{Q}$

c

e

$f$

m

TA

TN

$u$

VA

v

$\bar{u}$

$a, b, c, d, e$

Nutzungsgrad

Regressionsfunktionswert

Raumheizlast

Dichte, Raumgewicht

Stromkennzahl, Standardabweichung der Grundgesamtheit

relative Genauigkeit, relative Abweichung des Stichprobenmittelwertes vom Mittelwert der Grundgesamtheit

Temperatur

Leistung, Wärmeleistung

Konstante nach $\operatorname{ReCiPe}\left(\frac{10^{11}}{a}\right)$

Anlagenaufwandszahl, Eulersche Zahl

Faktor, Funktion

Masse

Amortisationsdauer

Nutzungsdauer einer Anlagenkomponente

spezifische Umweltleistung

Verlegeabstand von Fußbodenheizungsrohren

empirischer Variationskoeffizient

Mittelwert der simulierten spezifischen Umweltleistung

Annuität

$A$

Ein-/Auszahlungen, Ausgaben

$a$

Aufteilungsfaktor, Annuitätenfaktor

B

Bauhöhe, Barwert

$b$

preisdynamischer Barwertfaktor 
$D$

$d$

E

G

g
Durchmesser

Diskontierungssummenfaktor

Einzahlungen, Einnahmen, Erlöse

Gewichtungsfaktor

Faktor zur simulativen Gewichtung der Schadenskategorien für die Umweltwirkungsabschätzung

Investitionskosten

Konfidenzintervall

Charakterisierungsfaktor, Kapitalwert, Kosten

Korrekturfaktor, spezifische Kosten

Länge

Umfang der Grundgesamtheit oder Population

Stichprobenumfang, Ersatzhäufigkeit

Leistung, elektrische Leistung, Anteilsschätzer, Anteil der Werte unter- oder oberhalb des Mittelwertes einer Grundgesamtheit

Anzahl unabhängiger Variablen, Freiheitsgrade

Energie, Wärmebedarf

Abzinsungsfaktor, Zinsfaktor

Bestimmtheitsmaß

Widerstand, Wärmewiderstand, Restwert

Preisänderungsfaktor

standard error of regression, Standardregressionsfehler

sum of squares residual, Residuenquadratsumme

sum of squares, Abweichungsquadratsumme

Stärke, Wand- bzw. Materialstärke, Stichprobenstandardabweichung 
Betrachtungszeitraum

$T$

Typ, Heizkörpertyp, thermodynamische Temperatur

UI

Umweltleistungsindikator

UWI

Ökoeffizienzindikator

UW

Umweltwirkung, charakteristische Menge einer Umweltwirkungskategorie (Schadenskategorie)

U

Umweltleistung

V

Volumen

WI

Wirtschaftlichkeitsindikator

Wirtschaftlichkeit, Energie, Hilfsenergiebedarf

$X$

Elementarflussmenge

Y

Auslegungsgröße, Bezugsauslegungsgröße, empirischer Wert einer Zufallsgröße oder eines Merkmals

Zahl, Anzahl

Quantil, z-Wert, zentrale Wahrscheinlichkeit der Standardnormalverteilung

\section{Indizes}

$\lambda$

0

$\dot{Q}$

A

$B$

$f$

$H$
Wärmeleitfähigkeit

Investitionszeitpunkt

Leistung, Wärmeleistung, wärmeleistungsbezogen

Stromerzeugung, Ein-/Auszahlungen, Ausgaben

Barwert

Faktor, faktorbezogen

heating value, Energiegehalt 


$\begin{array}{ll}i, j, k & \text { laufender Zeiger } \\ K & \text { Kapitalwert } \\ Q & \text { Energie, Wärmebedarf } \\ t & \text { laufender Zeiger einer Periode } \\ \text { UI } & \text { Umweltleistungsindikator } \\ \text { UWI } & \text { Ökoeffizienzindikator } \\ U & \text { Umweltleistung } \\ u & \text { spezifische Umweltleistung } \\ \text { WI } & \text { Wirtschaftlichkeitsindikator }\end{array}$

A Anlagenkomponente

a annual, jährlich, außen

All Allokation, Zuordnung, Zuteilung

Ausl Auslegung

B Boden, Fußbodenbelag, Betrieb

b building energy use, Nutzenergiebedarf

betr. betriebsgebunden

C Carnot, Carnot-Wirkungsgrad, Carnot-Faktor

CHP combined heat and power, Kraft-Wärme-Kopplung

$\mathrm{CO}_{2} \quad$ Kohlenstoffdioxid

D Dimensionierung, Auslegung

Dä Dämmung, Isolierung

E Endenergieträger, Endenergie, Erlöse, Entsorgung

e elektrisch

ED Ecosystem Diversity, Ökosystemdiversität

EP Endpoint 
Er

Ers

f

$\mathrm{FBH}$

FW

G

$\mathrm{H}$

HE

$\mathrm{HH}$

$\mathrm{HL}$

HP

i

IN

Inst

K

k

KWK

L

$\mathrm{m}$

N

ne

o

oEr

oG

$\mathrm{p}$

$X X$
Erzeugung

Ersatz

End-, final, delivered

Fußbodenheizung

Fernwärme

Grund, Grundpreis, Gewichtung

Herstellung

Hilfsenergie

Human Health, menschliche Gesundheit

Heizlast

heating plant, Heizwerk

inferior (unterer), innen

Instandhaltung

Instandsetzung

kapitalgebunden

laufender Zeiger der Umweltschadenskategorie

Kraft-Wärme-Kopplung

Lüftungswärmeverluste

mittel, mittlere Temperatur der Wärmeeinspeisung

Bezugsnormal, Norm, Nennwert, Nutzung

netto

oberer

ohne Erzeugung

ohne Gewichtung

Primär-, primary 


\begin{tabular}{|c|c|}
\hline $\mathrm{PH}$ & Plattenheizkörper \\
\hline RA & Resource Availability, Ressourcenverfügbarkeit \\
\hline $\mathrm{ReCiPe}$ & ReCiPe-Methode \\
\hline Ref & Referenz \\
\hline S & Sonstige, Szenario \\
\hline s & superior (oberer), simulativ \\
\hline sonst. & sonstiges \\
\hline Sp & Speicherung \\
\hline SR & Stahlradiator \\
\hline $\mathrm{T}$ & Transmissionswärmeverluste \\
\hline U & Umgebung \\
\hline $\mathrm{u}$ & unterer \\
\hline Ue & Übergabe \\
\hline V & bedarfsgebunden, früher: verbrauchsgebunden \\
\hline Ve & Verteilung \\
\hline verbr. & verbrauchsgebunden \\
\hline W & Warmwasserbereitung, Wert \\
\hline W.Insp & Wartung und Inspektion \\
\hline WE & Wärmeerzeuger \\
\hline Äq. & Äquivalente \\
\hline
\end{tabular}


https://doi.org/10.51202/9783186006233-

Generiert durch IP '172.22.53.54', am 26.04.2023, 04:17:12.

Das Erstellen und Weitergeben von Kopien dieses PDFs ist nicht zulässig. 